\title{
Current-Level Discrimination in the Context of Interleaved, Multichannel Stimulation in Cochlear Implants: Effects of Number of Stimulated Electrodes, Pulse Rate, and Electrode Separation
}

\author{
Ward R. Drennan ${ }^{1,2}$ and Bryan E. Pfingst ${ }^{1}$ \\ ${ }^{1}$ Kresge Hearing Research Institute, University of Michigan, Ann Arbor, MI 48109-0506, USA \\ ${ }^{2}$ VM Bloedel Hearing Research Center, University of Washington, Box 357923, Seattle, WA 98195-7923, USA
}

Received: 25 January 2006; Accepted: 11 May 2006; Online publication: 21 June 2006

\begin{abstract}
The ability of cochlear implantees to detect an increment in current level at one of many stimulated electrodes was investigated. Such changes in the electric profile provide information for cochlear implantees to discriminate numerous sounds, especially vowels. In Experiment 1, sensitivity to increases in current level at one stimulation site in the electric profile decreased as the number of stimulated electrodes increased. This outcome was most likely a result of decreased stimulus levels at individual electrodes that were required to retain a comfortable loudness when the number of active electrodes was increased. Experiment 2 investigated the effects of pulse rate and separation between stimulation sites when the levels in percent of dynamic range and number of stimulated electrodes were held constant. The effect of pulse rate and electrode separation varied among listeners. The sensitivity of 6 of 9 listeners was best at the pulse rate that they used clinically. This might have been the result of adaptation to the clinical pulse rate, or listeners might have chosen their inherently best pulse rate during the clinical fitting.
\end{abstract}

Keywords: cochlear prosthesis, intensity discrimination, complex sound

Correspondence to: Ward R. Drennan · VM Bloedel Hearing Research Center - University of Washington - Box 357923, Seattle, WA 98195-7923, USA. Telephone: +1-206-8971848; fax: +1-2066161828; email: drennan@u.washington.edu

\section{INTRODUCTION}

Perceptions of changes in current level at individual electrodes are critical for many aspects of cochlear implant function. The perception of the current levelvs.-place profile corresponding to the acoustic spectral profile of the acoustic signal depends on the ability to discriminate the current level. These differences determine vowel identity, musical timbre, and spatial location. The perception of temporal amplitude modulations corresponding to the temporal envelope of the acoustic waveform will also be influenced by the ability of listeners to perceive changes in current level.

Studies by Nelson et al. (1996) and Drennan and Pfingst (2005) investigated current-level discrimination ability in cochlear implantees at single stimulation sites when no other electrodes were stimulated. However, in common sound processing schemes, such as continuous interleaved sampling (CIS), SPEAK, and ACE (Wilson et al. 1991; Skinner et al. 2002), multiple electrodes are stimulated. Thus, understanding how well implantees can hear changes in current level within the context of interleaved stimulation is essential for the optimal design of cochlear implant processors.

Green (1988) described a series of experiments designed to measure the minimal discriminable difference in the acoustic spectrum in normal-hearing listeners. The approach typically involved measuring the ability of a listener to hear an increment in one of many simultaneous sinusoids. In the cochlear implant, a similar approach can be used in which an electric profile (current-level vs. place profile) is pre- 
sented to the listeners by using interleaved, multichannel stimulation. To create a change in the electric profile shape, the current level on one or more electrodes is increased.

The ability to detect a change in current on one of many stimulated electrodes, however, might be compromised by channel interactions. Channel interactions can result from three phenomena (McKay 2004): (1) summation of the electric fields from multiple electrodes, (2) stimulation of common nerve bundles by multiple electrodes, and (3) perceptual summation or interference effects. Using interleaved, multichannel stimulation, there is no summation of electric fields. However, masking and summation effects have been observed previously with nonsimultaneous pulses across electrodes (Tong and Clark 1986; Chatterjee and Shannon 1998; Boëx et al. 2003; de Balthasar et al. 2003). These effects decrease with increased interelectrode distance (Shannon 1983; Lim et al., 1989) and decreased time intervals between pulses (Chatterjee and Shannon 1998; Boëx et al. 2003; de Balthasar et al. 2003). Masking effects have been observed for electrode separations of up to $14 \mathrm{~mm}$ and time intervals of up to $10 \mathrm{~ms}$. Faster pulse rates might lead to decreased sensitivity because of refractory effects, and closer electrode separation could increase these effects. There could be relatively small changes in the excitation pattern when an increment in current is added to a single electrode, thereby reducing sensitivity to the change. These observations concerning channel interactions led to the development of two hypotheses that we tested in Experiments 1 and 2, respectively-in the context of interleaved, multichannel stimulation: (1) sensitivity to changes in current level at a single stimulation site decreases as the number of other stimulated sites increases; and 2) single-site current-level discrimination ability decreases with faster pulse rates and decreased distances between stimulation sites.

\section{METHODS}

\section{Listeners}

Listeners who were implanted with Nucleus ${ }^{\circledR} 24 \mathrm{R}(\mathrm{CS})$ ContourTM $(N=6)$ or $24 \mathrm{M}(N=3)$ cochlear implants participated in this study. All listeners had full implant insertion according to the surgical records, and had a minimum of 6 months of experience with an activated implant. All listeners were postlingually deafened. Details of the listeners' characteristics are shown in Table 1. Listeners were paid to participate and reimbursed for lodging and travel expenses. All studies were reviewed and approved by the University of Michigan Medical School Institutional Review Board.

\section{Stimuli}

Symmetric biphasic pulses were presented to the listeners. The stimuli were $500 \mathrm{~ms}$ in duration. They were presented by using a monopolar (MP1 + 2) electrode configuration in which one stimulated electrode was within the cochlea and two return electrodes were outside the cochlea [(1) the plate electrode and (2) the ball electrode]. An interleaved stimulation strategy was employed when multiple electrodes were active. Individual biphasic pulses were equally spaced in time for each electric profile. Pulse trains were swept from apex to base. Table 2 summarizes the conditions tested and methods used, which are described in detail below.

Experiment 1. The independent variable in this experiment was the number of stimulated electrodes. Stimuli with 1, 3, 7, 11, and 21 stimulated electrodes were presented. For all five electric profiles in the initial portion of Experiment 1, the pulse rate was 250 pulses per second per channel (pps/channel), where a channel refers to the stimulus input to one electrode. The pulse phase duration was $75 \mu$ s and

TABLE 1

\begin{tabular}{|c|c|c|c|c|c|c|c|}
\hline \multicolumn{8}{|c|}{ Listener characteristics } \\
\hline Listener & $\begin{array}{l}\text { Implant } \\
\text { Type }\end{array}$ & $\begin{array}{l}\text { Age at onset } \\
\text { of profound } \\
\text { deafness }\end{array}$ & $\begin{array}{l}\text { Duration } \\
\text { of deafness } \\
\text { in years }\end{array}$ & $\begin{array}{l}\text { Duration } \\
\text { of implant } \\
\text { use in years }\end{array}$ & $\begin{array}{l}\text { Etiology } \\
\text { of deafness }\end{array}$ & $\begin{array}{l}\text { Clinical pulse } \\
\text { rate (pps/channel) }\end{array}$ & $\begin{array}{c}\text { Speech processor } \\
\text { strategy }\end{array}$ \\
\hline L1 & $24 \mathrm{R}(\mathrm{CS})$ & 28 & 0.5 & 3 & Unknown & 1200 & ACE \\
\hline L2 & $24 \mathrm{R}(\mathrm{CS})$ & 36 & 30 & 4 & Familial & 250 & SPEAK \\
\hline L3 & $24 \mathrm{R}(\mathrm{CS})$ & 22 & 13 & $17^{\mathrm{a}}$ & Progressive & 1200 & $\mathrm{ACE}$ \\
\hline L4 & $24 \mathrm{R}(\mathrm{CS})$ & 44 & 1 & 2.5 & Trauma & 900 & ACE \\
\hline L5 & $24 M$ & 42 & 6 & 6.5 & Trauma & 250 & SPEAK \\
\hline L6 & $24 \mathrm{R}(\mathrm{CS})$ & 62 & 2 & 1.5 & Familial & 900 & ACE \\
\hline L7 & $24 \mathrm{M}$ & 37 & 0.5 & 6 & Familial & 250 & SPEAK \\
\hline L8 & $24 M$ & 50 & 1 & 6 & Progressive & 250 & SPEAK \\
\hline L9 & $24 \mathrm{R}(\mathrm{CS})$ & 68 & 5 & 2 & Familial & 900 & $\mathrm{ACE}$ \\
\hline
\end{tabular}

aL3 was reimplanted 4 years prior to the study. Those described with "Familial" etiology have a family history of hearing loss, so a genetic cause is presumed. The "duration of deafness" is the self-reported time after which hearing aids no longer provided any benefit to the users. 
TABLE 2

Stimuli and methods summary

Experiment 1

Experiment 2

\begin{tabular}{|c|c|c|}
\hline $\mathrm{T}$ and $\mathrm{C}$ measurements & \multicolumn{2}{|c|}{$\begin{array}{l}\text { Measured on individual electrodes using the pulse rate over all electrodes from } \\
\text { each condition. }\end{array}$} \\
\hline Loudness & Matched with adjustment & $\begin{array}{l}\text { Set to } 70 \% \text { dynamic range; } \\
\text { near loudness equality }\end{array}$ \\
\hline Pulse rates & 250 and 159 pps/channel & $\begin{array}{l}250,720,900,1200, \\
1800 \mathrm{pps} / \text { channel }\end{array}$ \\
\hline Pulse width / gap & $75 \mu \mathrm{s} / 24 \mu \mathrm{s}$ & $25 \mu \mathrm{s} / 8 \mu \mathrm{s}$ \\
\hline Number of electrodes & $1,3,7,11,21$ & 7 \\
\hline Electrode separation & Multiple separations (see text) & $\begin{array}{l}\text { Neighboring electrodes, } \\
\text { every other electrode }\end{array}$ \\
\hline $\begin{array}{l}\text { Total reversals/ Reversals } \\
\text { in threshold calculation }\end{array}$ & $14 / 8$ & $16 / 10$ \\
\hline
\end{tabular}

All conditions were repeated 10 times and used a modified 2AFC procedure with two-down, one-up adaptive tracking. Details are provided in the text.

the interphase gap was $24 \mu \mathrm{s}$. A subsequent portion of Experiment 1 was completed by using 11 and 21 stimulated electrodes with a pulse rate of $159 \mathrm{pps} /$ channel. Electrode 13 was selected as the central target electrode. The current level through electrode 13 was incremented for all electric profiles. The current level of the other electrodes remained constant. In the single-electrode case, electrode 13 was used. For the case with three stimulated electrodes, electrodes 5, 13, and 21 were used. For the case with seven stimulated electrodes, electrodes 4, 7, 10, 13, 16,19 , and 21 were used. For the case with 11 active electrodes, odd-numbered electrodes from 1 to 21 were used. All electrodes (1-21) were used in the 21active-electrode case. The pulse rate per electrode remained constant such that the pulse rates over all electrodes for the 3-, 7-, 11-, and 21- active-electrode cases were $750,1750,2750$, and 5250 pps, respectively. In the subsequent portion of this experiment, in which a pulse rate of $159 \mathrm{pps} /$ channel was used with the 11- and 21-component electric profiles, the overall pulse rates were 1749 and 3339 pps, respectively. The pulse rate of $159 \mathrm{pps} / \mathrm{channel}$ was selected so that the time interval between individual pulses for seven stimulated electrodes at $250 \mathrm{pps} / \mathrm{channel}$ and for 11 stimulated electrodes at $159 \mathrm{pps} / \mathrm{channel}$ was nearly the same. The experiment with 159 pps/ channel was conducted to investigate possible refractory effects and the effect of pulse rate on electricprofile discrimination ability.

Experiment 2. The independent variables in this experiment were pulse rate and the separation between stimulated electrodes. Five pulse rates were used: 250, 720, 900, 1200, and 1800 pps/channel. The pulse rates were among the rates that can be chosen clinically, using the Nucleus 24 R(CS) and 24M implants. Two 7-electrode profiles were used. These were labeled "dense" and "spaced." The "dense" profile engaged all stimulation sites from 10 to 16 . The "spaced" profile used odd-numbered electrodes from 7 to 19. The pulse phase durations and interphase gaps were 25 and $8 \mu$ s, respectively. These were clinically common pulse durations.

\section{Threshold and comfortable loudness levels}

Threshold ( $\mathrm{T}$ ) and maximum comfortable loudness (C) levels were measured by using the method of adjustment. Using in-house software, listeners used a computer mouse to adjust the level on an individual electrode until it was just audible. Then, the listeners adjusted the current level on the same electrode upward until it reached the maximum level at which they felt they could listen for hours without discomfort.

Experiment 1. In the clinic, $\mathrm{T}$ and $\mathrm{C}$ levels are typically measured on each electrode individually, by using a given pulse rate per channel. However, when multiple electrodes are stimulated, summation effects occur. If all electrodes were active at their individual $C$ levels, the overall loudness of the multielectrode stimulation would be well above the $\mathrm{C}$ level and therefore uncomfortably loud. Clinically, all the $\mathrm{T}$ and $\mathrm{C}$ levels are adjusted downward so that the overall level is amenable to the user. Loudness levels on a single electrode are much closer to loudness levels with multiple electrodes if the overall pulse rate for multiple electrodes is used to measure $\mathrm{T}$ and $\mathrm{C}$ on a single electrode (McKay et al. 2003). Therefore, for the electrodes used in the 1-, 3-, 7-, 11-, and 21active-electrode conditions, $\mathrm{T}$ and $\mathrm{C}$ levels for individual electrodes were determined by using 250, 750, 1750,2750 , and 5250 pps, respectively. To determine the $\mathrm{T}$ and $\mathrm{C}$ levels for the 159-pps conditions, $\mathrm{T}$ and $\mathrm{C}$ levels were measured for the electrodes used for 11 and 21 stimulated electrodes at 1749 and 3339 pps, respectively. 
Experiment 2. $\mathrm{T}$ and $\mathrm{C}$ levels for individual electrodes were measured by using the overall pulse rates of the seven-electrode conditions: 1750, 5040, 6300, 8400 , and 12,600 pps.

\section{Level of presentation}

Experiment 1. All profiles were matched in loudness to that of the seven-electrode profile in which each component was set to $70 \%$ of the dynamic range based on a linear microamp scale. This procedure was carried out to control for overall loudness and because implantees typically listen to sounds (especially speech) at about this level. Listeners heard alternating 500-ms presentations of the seven-component electric profile followed by one of the other profiles. The duration of each stimulus and the interval between stimuli was $500 \mathrm{~ms}$. Listeners used in-house software to adjust the level of the second stimulus until its loudness matched that of the first. The seven-electrode profile set at $70 \%$ of the dynamic range was presented in the first interval. During adjustment, listeners clicked on a mouse to increase or decrease the stimulus level. With each mouse click, the level in the second interval changed by $1 \%$ of the dynamic range on all stimulated electrodes. Levels were rounded to the nearest clinical level unit. For example, in a given block, listeners might hear the seven-electrode profile followed by the 11-electrode profile in alternation. The listener would adjust the level of the 11-electrode profile up or down until the overall level matched the loudness of the sevenelectrode profile. The loudness matching procedure would then start again with a different profile in the second interval. All four electric profiles were matched in loudness to the seven-active-electrode profile. The matching procedure was repeated four times for each profile. For each listener, the average matched level was used as the level of the "standard" electric profile in Experiment 1.

Experiment 2. McKay et al. (2003) predict that stimulation of $N$ active electrodes of equal loudness will produce the same loudness as stimulation of one electrode using $N$ times the pulse rate. We set the level of each electrode to $70 \%$ of the dynamic range measured by using single electrodes at the overall pulse rate. In a pilot study using two listeners, we implemented the loudness matching procedure for electric profile with all pulse rates, but we found that no adjustments were needed. Thus, McKay et al.'s model predictions were borne out empirically, and, therefore, loudness matching was judged to be unnecessary in Experiment 2. The presentation levels were set to $70 \%$ of the dynamic range on each electrode using the overall pulse rate.

\section{Procedure}

A modified 2AFC procedure was used with flanking stimuli such that there were four presentations on each trial. The stimulus durations and interstimulus intervals were $500 \mathrm{~ms}$ each. The first and last presentations were the standard profiles. One of the middle two presentations contained an increment in current at electrode site 13 . The minimum detectable increments were determined by using a two-down, one-up adaptive tracking method converging on $70.7 \%$ correct (Levitt 1971). The step size was one clinical level step equal to $0.176 \mathrm{~dB}$ of current in microamps equivalent to a $2.046 \%$ increase in current level. In the adaptive tracking for Experiment 1, 14 reversals were completed and the difference limen (DL) was calculated as the mean of the values at last eight reversals. In Experiment 2, 16 reversals were completed, and DL was calculated as the mean of the values at the last 10 reversals. Each complete series of reversals constituted a tracking history.

Electric profiles were presented in blocks defined as sets of 10 tracking histories. Ten DL measures (independent tracking histories) were completed in each condition. In each block of Experiment 1, two repetitions of each profile were presented in a random order. Five blocks were completed. In Experiment 2, 10 blocks of 10 tracking histories were completed. Each block contained profiles of the five pulse rates and the two electrode separations. Blocks required 60-90 min to complete.

In Experiment 1, for all but one listener, the 11and 21-electrode profiles were presented at a pulse rate of $159 \mathrm{pps} / \mathrm{channel}$ after testing with profiles of $250 \mathrm{pps} /$ channel was completed. The tracking histories were presented in an alternating order such that listeners heard the $250 \mathrm{pps} /$ channel stimulus in the first tracking history and heard the $159 \mathrm{pps} /$ channel stimulus in the second tracking history in order, repeated 10 times. Two blocks of 10 tracking histories were completed such that DLs were obtained for each profile 10 times.

For analysis, the DL was converted from the clinical level steps into linear microamps. This was performed by using the conversion formula $I=F \times$ $10 \times 175^{(\mathrm{CL} / 255)}$, where $\mathrm{F}$ is the calibrating variable that had a unique value for each implant stimulator based on the maximum average current output across all electrodes as measured by the Cochlear Corporation, and CL is the clinical level step. DLs were calculated as Weber fractions $(W f)$, which were determined by using the equations described by Pfingst et al. (1983) and Nelson et al. (1996) in which $W f=2 \Delta I / I+(\Delta I / I) 2$. This Weber fraction is a power ratio. Data were plotted as $W f$ (in $\mathrm{dB}$ ) equal to $10 \log W f$. 


\section{Equipment}

All listeners used a common, laboratory-owned Sprin $\mathrm{t}^{\circledR}$ processor (Cochlear Corporation) during the experiment. Sequences of frames were created and sent to the SPrint ${ }^{\mathbb{B}}$ processor by using the Nucleus Implant Communicator ${ }^{\circledR}$ (v. 3.7) software libraries. The software for the experiment was run on an IBM-compatible computer. Communication with the Sprint processor was accomplished by using an IF5 ISA card and a Processor Control Interface (PCI) from Cochlear Corporation.

\section{RESULTS}

\section{Experiment 1: Effects of number of electrodes}

Figure 1 shows the results for six individual listeners, each in a separate plot. Data from the 250 and 159 pps/ channel conditions are shown. In the $250 \mathrm{pps} /$ channel condition, sensitivity to changes in the electric profile decreased (i.e., DLs increased) as the number of stimulated electrodes increased for all listeners. The extent of the effect was highly variable among listeners. The data for the $250 \mathrm{pps} /$ channel condition were analyzed by using a general linear regression model taking into account individual differences and the correlation among repeated measures. The linear relationship between number

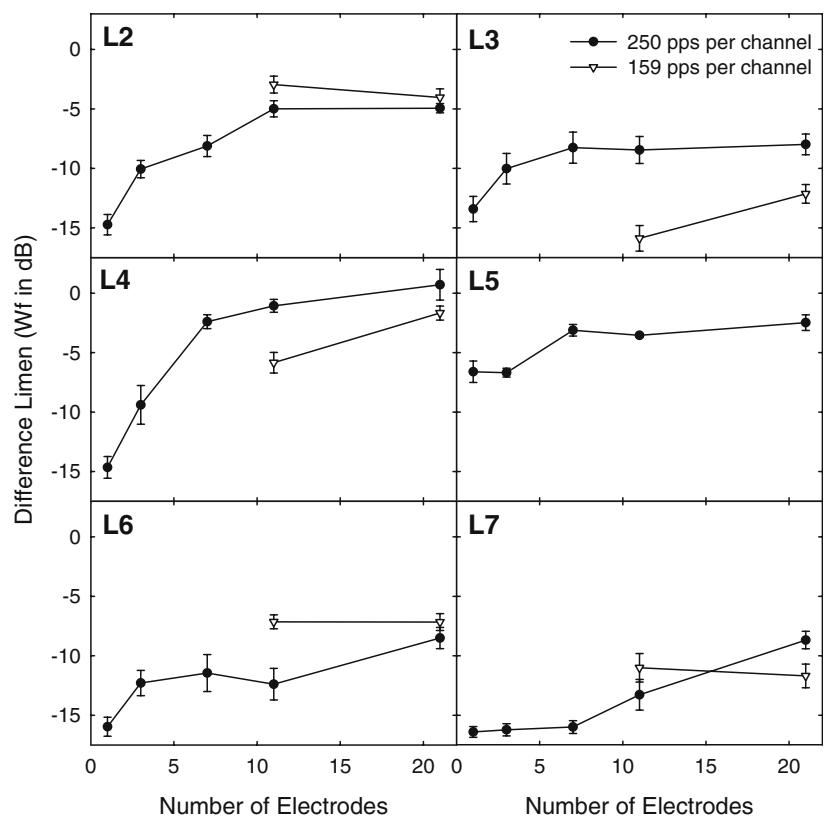

FIG. 1. The electric profile difference limen (DL) in Weber fractions $(W f$; in $\mathrm{dB}$ ) is shown as a function of the number of stimulated electrodes for each individual listener. Data are shown for 250 and 159 pulses per second per channel. Error bars show 1 standard error. Only six listeners participated. of electrodes and DL was found to be positive and statistically significant $(\beta=0.370, p=0.001)$. The regression coefficient $\beta$ indicated that, on average, Weber fractions increased by 0.37 for each active electrode added. No effects of time (learning) were observed.

Experiment 2: Effects of pulse rate and separation of stimulated electrodes

Figure 2 shows the results for the nine listeners who participated in Experiment 2. The data were variable among listeners. Individual listeners had best sensitivity at various pulse rates. Statistical analysis was performed by using a linear mixed model (Gueorguieva and Krystal 2004). A repeated-measures ANOVA was not appropriate given the variability among listeners and curvilinear functions of pulse rate. A quadratic term was included in the mixed model to account for curvilinear functions. Three listeners had slightly better sensitivity to increments in the spaced electric profile (filled circles) than to increments in the dense electric profile (open circles). The mean difference for all listeners was $0.96 \mathrm{~dB}$. The effect of electrode separation was significant $\left(F_{1,853}=51.437, p<0.001\right)$. No significant fixed effect of pulse rate on sensitivity to changes in current level was found in this analysis. No significant interactions were observed, and no effects of time of testing (learning) were observed. The arrows in Figure 2 show the listeners' clinical pulse rates (i.e., the pulse rates assigned in the clinic and used in the processors that they used daily). Interestingly, all listeners who used the SPEAK speech processing strategy, using a pulse rate of $250 \mathrm{pps} /$ channel (L2, L5, L7, and L8), had best sensitivity at $250 \mathrm{pps} /$ channel. For six of nine listeners (L2, L4, L5, L6, L7, and L8), the best electric profile sensitivity matched their clinical pulse rate.

\section{DISCUSSION}

\section{Experiment 1}

Sensitivity to current-level increases on a single electrode decreased as the number of active electrodes increased, consistent with our hypothesis. This result indicates that single-electrode current-level discrimination ability is not representative of currentlevel discrimination ability of a single electrode in the context of multichannel interleaved stimulation when the single-electrode and multichannel stimuli are presented at similar loudnesses. However, the reasons for the decrease in sensitivity appeared to be different than those proposed in the Introduction. When matched for loudness, the absolute current levels on 


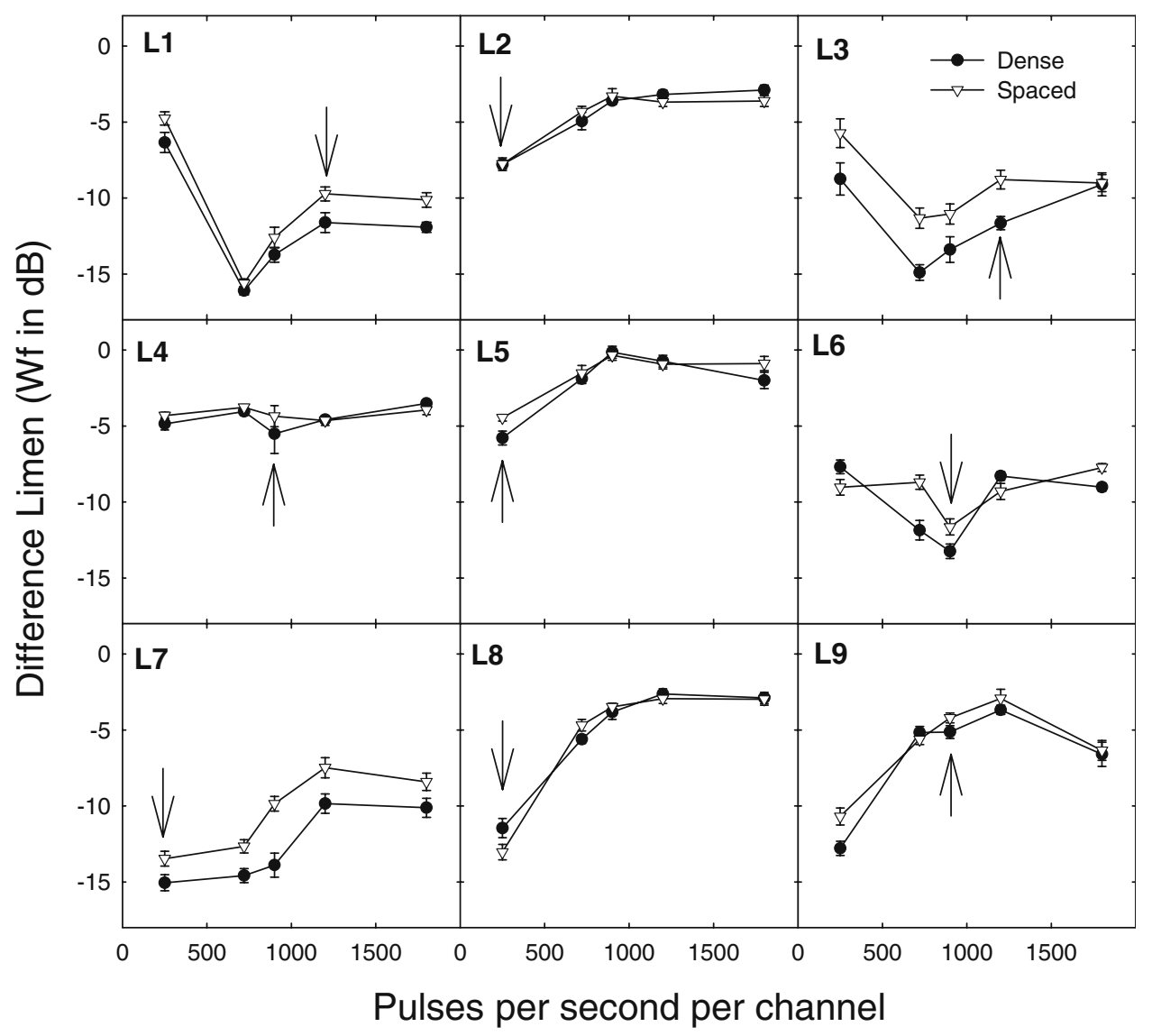

FIG. 2. The electric profile difference limen (DL) in Weber fractions $\left(W_{\mathrm{f}}\right.$; in $\left.\mathrm{dB}\right)$ is shown as a function of the pulse rate for each individual listener. Data are shown for the spaced (filled

all electrodes consistently decreased as more electrodes were added, consistent with the McKay et al. (2003) loudness model. As the number of electrodes increases, the relative contribution of the target component to overall loudness decreases. If listeners based a decision in this task on overall loudness changes, the model predicts that sensitivity would get worse. Additionally, in single-electrode current-level discrimination experiments, sensitivity usually decreases with decreases in current level (Nelson et al. 1996; Drennan and Pfingst 2005). Thus, the main effects of Experiment 1 appeared to be attributable to the decrease in current level of the target, and consequently a decrease in its contribution to overall loudness as the number of electrodes increased.

For analysis, we implemented McKay et al.'s (2003) loudness model. Although we did not collect loudness growth-vs.-current level data for our listeners, we predict results for Experiment 1 by using a known loudness-vs.-level curve from McKay et al. (2003), and assume that a loudness difference $(\Delta L)$ of 10 units is detectable in all cases. The model predicts loudness $(L)$ by summing the loudness of each individual circles) and dense (open circles) electric profiles. Error bars show 1 standard error. The arrows indicate the pulse rate that the listeners use clinically.

pulse within a 2-ms time window. The log of loudness is then given by the following equation:

$$
\log (L)=a * c+0.03 * b * e^{\left(c-c_{0}\right) / b}+k
$$

in which $a, b$, and $k$ are model parameters determined empirically; $c_{0}$ is a reference loudness for a pulse of $c_{0}$ clinical units (for the Cochlear Corporation implant); and $c$ is the clinical level of the stimulus for which we wish to predict the loudness. The loudness of a complex stimulus is then determined by summing the loudness for each pulse within a 2-ms time window. Using empirical values for S5 from McKay et al. $\left(a=0.016, c_{0}=185, b=10, k=-1.8719\right)$, we generated a table showing loudness for each integer clinical level from a loudness of 0 to a loudness of 100. It was assumed that the loudness of the singleelectrode stimulus was 100 , that the loudnesses of all multiple-electrode stimuli were also 100 (the loudnesses of the electric profiles were matched in Experiment 1), and that the overall loudness required to detect a difference in level was 110 . Given that McKay et al.'s loudness model assumes independent 
loudness contributions from individual pulses, we estimated the level of presentation of the single electrode for S5 by dividing the overall loudness by the number of stimulated electrodes. The clinical level units corresponding to single-electrode loudness, determined by using the table of loudness vs. clinical level, were 194, 191, 188, 183, and 175 clinical units for the 1-, 3-, 7-, 11-, and 21-component electrodes, respectively. These levels (decreasing with increasing numbers of electrodes) were typical of those observed in our study. The table was used to determine the number of clinical unit steps required to increase loudness by a minimum of 10 units. We converted the clinical unit steps to microamps by using the equation above, and subsequently calculated the $W f$ (in $\mathrm{dB}$ ) required to produce a loudness increment of 10 units for each profile. The result is shown in Figure 3. This prediction parallels the result of Experiment 1 . The effects in Experiment 1 appear to be indirectly attributable to summative channel interaction effects. Loudness from individual electrodes combine, thereby reducing the contribution of each single electrode to the whole and making discrimination of currentlevel increase more difficult in the presence of interleaved multichannel stimulation at a comfortable loudness level.

Masking effects might have contributed to the decrease in sensitivity, but given that the loudness model fits the data well, and it does not incorporate any refractory-type masking effects, we conclude that masking effects were slight or nonexistent. We had initially explored the possibility that temporal effects (forward masking) might have influenced the effect of number of electrodes. This was done by decreasing the pulse rate: i.e., the 11- and 21-component profiles were retested by using $159 \mathrm{pps} /$ channel and the results were compared to those for stimulation at

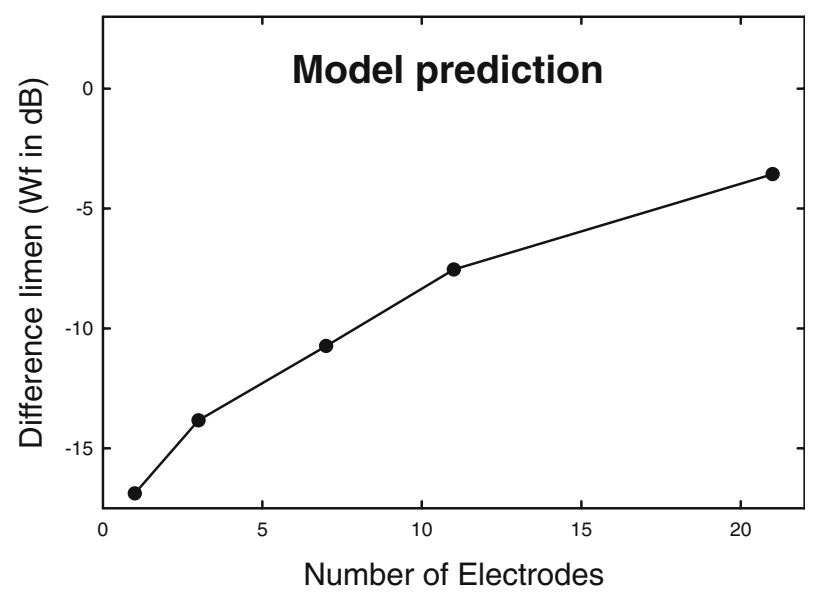

FIG. 3. Results from the implementation of the McKay et al. (2003) loudness model are similar to the empirical results from Experiment 1.
250 pps/channel. The results, shown in Figure 1, were variable. Some listeners showed improved sensitivity at $159 \mathrm{pps} / \mathrm{channel}$ and others showed poorer sensitivity. Consistent with the predictions of the loudness model, this finding suggested that channel interaction resulting from higher pulse rates was not a factor. However, the pulse rates used in this test were slower than normal clinical rates. McKay et al. (2003) noted that at higher pulse rates, refractory effects might come into play. Experiment 2 investigated the pulse rate effects with higher rates that are commonly used clinically.

\section{Experiment 2}

Results of Experiment 2 were not consistent with the hypothesis that higher pulse rates lead to decreased sensitivity. The electrode separation results suggest that more channel interaction might have been present with minimal electrode separation, but the effect was small and only observed in one-third of the implantees. Furthermore, no interaction was observed between electrode separation and pulse rate. We might have expected that the effect of separation would increase with pulse rate, but this was not observed. Therefore, the expected large effects of increased channel interaction were not observed at the pulse rates, electrode separations, and levels used in this study. Given previous results from Boëx et al. (2003) showing channel interactions at up to $14 \mathrm{~mm}$ electrode separation and $10 \mathrm{~ms}$ temporal separation, we expected more effects related to channel interaction; however, these previous studies on forward masking involved detection of a single low-level pulse. Our study used a discrimination task with pulse trains at suprathreshold levels. The current level of the target component then was well above the detection threshold and above the level of the standard (comparison) component. It appears that masking effects on this task were minimal. Channel interactions might have indirectly contributed as loudness summation effects, rather than as masking effects. That is, the neural responses from multiple electrodes add up, creating greater loudness, requiring lower currents on individual electrodes to achieve a comfortable listening level, and resulting in poorer current-level discrimination ability on individual stimulation sites.

The loudness model used to predict results in Experiment 1 was also applied to Experiment 2. The absolute levels of the target component in Experiment 2 decreased as the pulse rates increased, as the model predicted. However, the predicted DLs did not always decrease, because the number of times the incremented target component fell within the 2-ms 
time window also increased with increasing pulse rate. Assuming that the electric profiles all had a loudness of 100 and were discriminable at loudness 110 , the predicted Weber fractions in $\mathrm{dB}$ were -3.5 , $-2.5,-1.2,-1.5$, and -2.1 for $250,720,900,1200$, and $1800 \mathrm{pps} /$ channel, respectively. This predicted sensitivity was usually worse than, and did not correspond well with the observed results, suggesting that factors other than loudness were contributing to the outcome. Pulse-rate familiarity was likely to be a contributing factor.

The listeners in Experiment 2 commonly had best sensitivity at a pulse rate that matched the pulse rate used daily in their clinical device. Listeners might have adapted to the pulse rate they used clinically, and became more tuned to changes in current level at that pulse rate. Alternatively, listeners might have had an inherent best pulse rate. Clinical pulse rates are usually adjusted by the audiologist to suit the patient. Typically, ACE at $900 \mathrm{pps} /$ channel is the default selection, and if the users are not happy with that, the audiologist will try SPEAK at $250 \mathrm{pps} /$ channel or a different pulse rate with ACE (720, 1200 , or $1800 \mathrm{pps} /$ channel). Some implantees might have had physiological characteristics such that they were most sensitive to the electric profile shape at $250 \mathrm{pps} / \mathrm{channel}$ and therefore preferred to use the SPEAK strategy. In animal studies, Shepherd and colleagues (2004) have demonstrated that refractory periods can be longer for subjects that have been deaf for a longer period of time. Longer durations of deafness generally result in nerve degeneration. Similar degenerative variability exists in humans related to a large variety of etiologies and durations of deafness (Nadol et al. 1989; Nadol 1997). Such variability in the nerve health could have caused the across-listener variation in effects of pulse rate observed in our experiments.

Similar pulse-rate-dependent variability in performance has been observed in studies of speech understanding. For example, Vandali et al. (2000) found wide variability in the effects of pulse rate on open-set speech recognition ability for five implantees listening to speech in multitalker noise. Loizou et al. (2000) reported variability among listeners in speech understanding dependent upon pulse rates and pulse durations. Holden et al. (2002) found, by using pulse rates of 729 and 1800 pps, that some listeners had better speech understanding in noise for the higher pulse rate, whereas others performed better with lower pulse rates. The results from Experiment 2 demonstrate that this across-listener variability in the effects of pulse rate extends to a simpler psychophysical task, which suggests a mechanism underlying the variability in the speech tasks.

\section{ACKNOWLEDGMENTS}

The authors are grateful for the assistance of software engineer Thyagarajan Sadasiwan, statistician Brady West, research assistants Tricia Hayes and Cathy Thompson, and our dedicated listeners. Marie Leonard and two anonymous reviewers provided helpful comments on previous versions of this manuscript. This work was supported by NIH/ NIDCD grants F32-DC005893, R01-DC03808, and the KHRI Computing and Electronics core facilities (P30 DC05188).

\section{REFERENCES}

Bö̇x C, Kos M-I, Pelizzone M. Forward masking in different cochlear implant systems. J. Acoust. Soc. Am. 114:2058-2065, 2003.

Chatterjee M, Shannon RV. Forward masked excitation patterns in multielectrode electrical stimulation. J. Acoust. Soc. Am. 103:2565-2572, 1998.

de Balthasar C, Boëx C, Cosendai G, Valentini G, Sigrist A, Plizzone M. Channel interactions with high-rate biphasic electrical stimulation in cochlear implant subjects. Hear. Res. 182:77-87, 2003.

Drennan WR, Pfingst BE. Current-level discrimination using bipolar and monopolar electrode configurations in cochlear implants. Hear. Res. 202:170-179, 2005.

Green DM. Profile Analysis. Oxford University Press, NY, 1988.

Gueorguieva R, Krystal JH. Move over ANOVA. Arch. Gen. Psychiatry 61:310-317, 2004.

Holden LK, Skinner MW, Holden TA. Effects of stimulation rate with the Nucleus 24 ACE speech coding strategy. Ear. Hear. 23:463-476, 2002.

LevitT H. Transformed up-down methods in psychoacoustics. J. Acoust. Soc. Am. 49:467-477, 1971.

Lim HH, Tong YC, Clark GM. Forward masking patterns produced by intracochlear electrical stimulation of one and two electrode pairs in the human cochlea. J. Acoust. Soc. Am. 86:971-980, 1989.

Loizou PC, Poroy O, Dorman M. The effect of parametric variations of cochlear implant processors on speech understanding $\mathrm{J}$. Acoust. Soc. Am. 108:790-802, 2000.

McKAY CM. Psychophysics and electrical stimulation. In: Zeng FG, Popper AN, and Fay RR (eds) Cochlear Implants: Auditory Prostheses and Electric Hearing. Springer-Verlag, New York, pp 286-333, 2004.

McKay CM, Henshall KR, Farrell RJ, McDermott HJ. A practical method of predicting the loudness of complex electrical stimuli. J. Acoust. Soc. Am. 113:2054-2063, 2003.

NADOL JB JR. Patterns of neural degeneration in the human cochlear and auditory nerve: implications for cochlear implantation. Otolaryngol. Head Neck Surg. 117:220-228, 1997.

NAdol JB JR., Young Y-S, GLYNn RJ. Survival of spiral ganglion cells in profound hearing loss: implication for cochlear implantation. Ann. Otol. Rhinol. Laryngol. 98:411-416, 1989.

Nelson DA, Schmitz JL, Donaldson GS, Viemeister Ne, Javel E. Intensity discrimination as a function of stimulus level with electric stimulation. J. Acoust. Soc. Am. 100:2393-2414, 1996.

Pfingst BE, Burnett PA, Sutton D. Intensity discrimination with cochlear implants. J. Acoust. Soc. Am. 73:1283-1292, 1983.

SHANNON RV. Multichannel electrical stimulation of the auditory nerve in man. I. Basic psychophysics. Hear. Res. 11:157-189, 1983. 
Shepherd RK, Roberts LA, Paolini AG. Long-term sensorineural hearing loss induces functional changes in the rat auditory nerve. Eur. J. Neurosci. 20:3131-3140, 2004.

Skinner MW, Arndt PL, Staller SJ. Nucleus 24 advanced encoder conversion study: performance vs preference. Ear Hear. 23: 2S-25S, 2002.

TONG YC, CLARK GM. Loudness summation, masking and temporal interaction for sensations produced by electric stimulation of two sites in the human cochlea. J. Acoust. Soc. Am. 79: 1958-1966, 1986.

Vandali AE, Whitford LA, Plant KL, Clark GM. Speech perception as a function of electrical stimulation rate: using the Nucleus 24 cochlear implant system. Ear Hear. 21:608-624, 2000.

Wilson BS, Finley CC, Lawson DT, Wolford RD, EdDington DK, Rabinowitz WM. Better speech recognition with cochlear implants. Nature 352(6332):236-238, 1991. 